\title{
Inspecting adaptive optics with at-wavelength wavefront metrology.
}

\author{
Krempaský J.a, Koch F.c, Vagovič P. ${ }^{\text {b,d }}$, Mikeš L. ${ }^{\text {d }}$, Jaggi A. ${ }^{a}$, Svetina C. ${ }^{a}$, Flechsig U. ${ }^{a}$, \\ Patthey L. ${ }^{a}$, Marathe S. ${ }^{\text {, }}$ Batey D. ${ }^{\text {f }}$, Cipiccia S. ${ }^{\mathrm{f}}$, Rau C. ${ }^{\mathrm{f}}$, Seiboth F. ${ }^{\mathrm{e}}$, Seaberg M. ${ }^{\mathrm{g}}$, David \\ C. ${ }^{\mathrm{a}}$, and Wagner U. H. ${ }^{\mathrm{a}}$

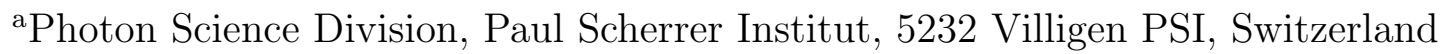 \\ ${ }^{\mathrm{b}}$ Center for Free-Electron Laser Science, DESY, Notkestrasse 85, 22607 Hamburg, Germany \\ ${ }^{\mathrm{c}}$ Laboratory for Micro and Nanotechnology, Paul Scherrer Institut, 5232 Villigen PSI, \\ Switzerland \\ dEuropean XFEL GmbH, Albert-Einstein-Ring 19, 22761 Hamburg, Germany \\ ${ }^{\text {e}}$ Deutsches Elektronen-Synchrotron DESY, Notkestr. 85, 22607 Hamburg, Germany

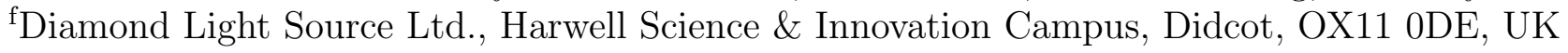 \\ 'Linac Coherent Light Source, SLAC National Accelerator Laboratory, 2575 Sand Hill Road, \\ Menlo Park, California 94025, USA.
}

\begin{abstract}
Preserving the coherence and wavefront of a diffraction limited x-ray beam from the source to the experiment poses stringent quality requirements on the production processes for X-ray optics. In the near future this will require on-line and in-situ at-wavelength metrology for both, free electron lasers and diffraction limited storage rings. A compact and easy to move X-ray grating interferometry (XGI) setup has been implemented by the Beamline Optics Group at PSI in order to characterize x-ray optical components by determining the aberrations from reconstructing the x-ray wavefront. The XGI setup was configured for measurements in the moiré mode and tested with focusing optic at Swiss Light Source, Diamond Light Source and LCLS. In this paper measurements on a bendable toroidal mirror, a zone plate, a single and a stack of beryllium compound refractive lenses (CRL) are presented. From these measurements the focal position and quality of the beam spot in terms of wavefront distortions are determined by analysing the phase-signal obtained from the XGI measurement. In addition, using a bendable toroidal mirror, we directly compare radius of curvature measurements obtained from XGI data with data from a long-trace profilometer, and compare the CRL wavefront distortions with data obtained by ptychography.
\end{abstract}

Keywords: X-ray grating interferometry;wavefront metrology; X-ray optics; XFELs.

\section{INTRODUCTION}

The need to preserve the coherence and wavefront of x-ray beams generated by the latest generation of synchrotrons and XFELs for state-of-the-art experiments requires increasingly sensitive diagnostic instrumentation, pushing the resolution and sensitivity towards the nanometer scale. Recently at-wavelength wavefront metrology has been gaining popularity especially due to in situ XGI single-shot wavefront metrology developed by Kayser et al. ${ }^{1}$ This is particularly relevant for wavefront monitoring of pulsed X-ray sources, whose highly coherent and brilliant light is focused down to sub $100 \mathrm{~nm}$ spots.

In this contribution we characterize the wavefront by means of a moiré X-ray interferometer. ${ }^{2,3}$ The instrument, deployed at the Swiss Light Source (SLS) at the X05DA Optics beamline, ${ }^{4}$ is schematically depicted in Fig. 1, the bottom figure shows the modular, compact, transportable and self-contained XGI setup: The moiré

Further author information: (Send correspondence to J.K.)

J.K.: E-mail: juraj.krempasky@psi.ch, Telephone: ++41 563105131

U.W.: E-mail: ulrich.wagner@psi.ch, Telephone: +41 563102179

Adaptive X-Ray Optics V, edited by Daniele Spiga, Hidekazu Mimura, Proc. of SPIE Vol. 10761, 107610D - (C) $2018 \mathrm{SPIE} \cdot$ CCC code: 0277-786X/18/\$18 - doi: 10.1117/12.2320532 


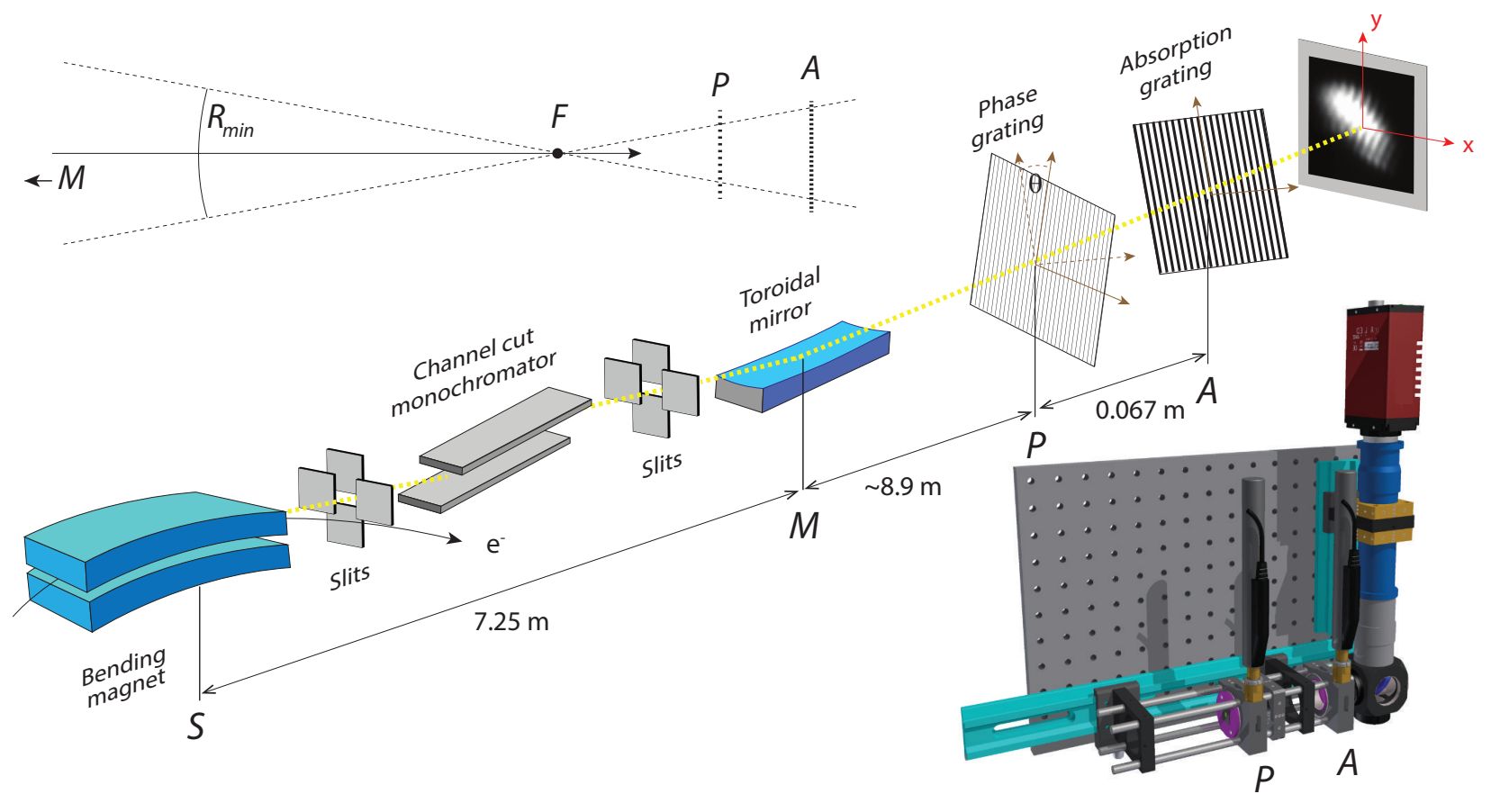

Figure 1. Schematic of the optical scheme of the SLS optics beamline (not to scale), with moiré XGI experimental setup. The phase grating $P$ is located near the nominal beam focus $F$ from the focusing toroidal mirror $M$. (top) Simplified layout indicating focus position displacement $F$ from mirror $M$, with respect to phase (absorption) grating $P(A)$, see text. (bottom) Schematic of the transportable and self-contained XGI setup.

XGI setup consists of an upstream $\pi$-shifting phase grating acting as a beam splitter, and a downstream absorption grating acting as a transmission mask, as shown in Fig. 1 as $P$ and $A$, respectively. The moiré fringes are the result of the superposition of the self-image of the first grating with the absorbing grating structure of the second grating. The interference pattern produced by the phase grating at the distance $P A$ where the second absorption grating is located, is magnified from the expected Talbot self-image by a magnification factor $M=\frac{F P+P A}{F P}$ (see Fig. 1 top). The fringe spacing depends on the relative angular alignment of the two gratings (rotation around the x-ray beam axis). The angle between the gratings can be tuned to generate tightly spaced fringes, thus achieving a high spatial resolution, or coarsely spaced fringes providing high phase sensitivity. ${ }^{3}$

The moiré fringes that are resolved using a 2D detector with a Ce-doped YAG screen (10 $\mathrm{mm}$ in diameter, 30 $\mu \mathrm{m}$ thickness) scintillation screen, located right behind the second grating and converts the x-ray fringe image into a visible light image. The image is observed with an infinity corrected long working distance microscope objective, which relays the moiré pattern onto a 14-bit GigE CCD camera $(1024 \times 1024$ pixels with a 5.5 micron size), controlled through EPICS with the areaDetector software. ${ }^{5}$ The overall effective pixelsize is 2.857 microns. A more detailed description of the optical setup with is described in Ref. 3.

In our previous studies we have been using divergent beams. ${ }^{3}$ In these studies we matched the grating pitch of the Talbot self-image generated with a 4 micron, $\pi$-shifting phase-grating, to an absorption grating with 2 micron pitch. However, if using focusing optics with different focal length, as depicted in Fig. 1, matching the self-image to the same absorption grating $A$ implies that the phase grating period needs to be adjusted. There is a certain leeway in producing moiré fringes for both divergent and focusing beams by optimizing the grating period, photon energy, Talbot distance $P A$, and associated fractional Talbot order. ${ }^{2}$ The limiting factor in our XGI setup is the maximum Talbot distance $P A$ of $\approx 19 \mathrm{~cm}$. Therefore we optimised the XGI parameters for a phase grating with a $3.75 \mu \mathrm{m}$ period according to Table 1, allowing us to investigate bendable mirror, zone plate and CRLs. The one-dimensional moiré XGI setup discussed below is well suited for such tasks, as it provides a quantitative measurement of the wavefront errors in one direction with high angular sensitivity, chosen to be 
along the optical element under test in vertical direction. We note that extensive characterization of CRLs with two-dimensional moiré XGI based on checkerboard structures, allowing full reconstruction of the lens profile with aberrations, are discussed in Refs. 6,7.

Table 1. Optimized moiré XGI setup parameters.

\begin{tabular}{|l|l|l|l|}
\hline Beamline & Photon energy & Talbot distance & Fractional Talbot order \\
\hline X05DA Optics bml. SLS & $8.3 \mathrm{keV}$ & $6.7 \mathrm{~cm}$ & 5 \\
\hline ID13 Coherence bml. DLS & $9.7 \mathrm{keV}$ & $17.2 \mathrm{~cm}$ & 11 \\
\hline XPP bml. SLAC & $9.45 \mathrm{keV}$ & $15.7 \mathrm{~cm}$ & 11 \\
\hline
\end{tabular}

\section{WAVEFRONT CHARACTERIZATION WITH MOIRÉ INTERFEROMETRY}
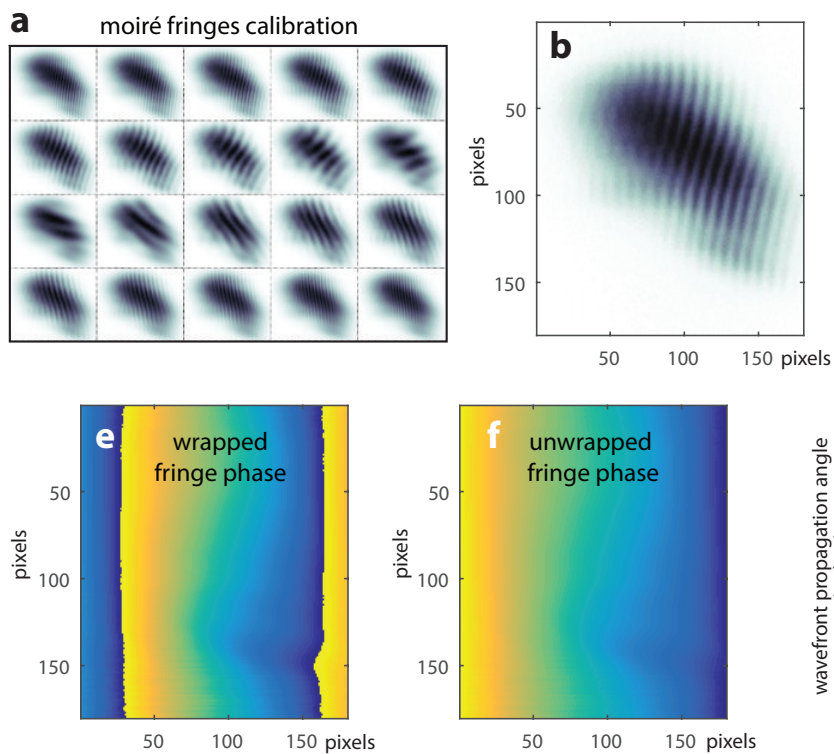

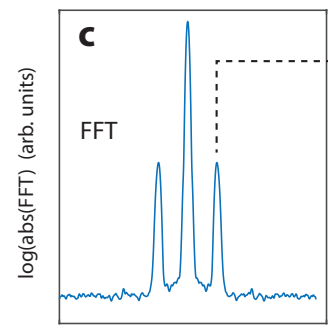

frequency (arb.units)

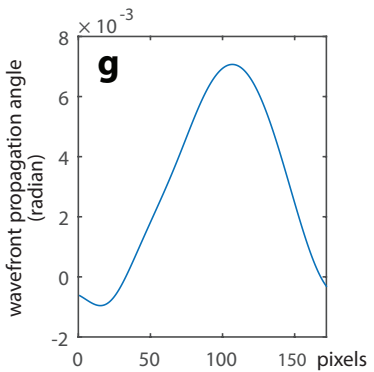

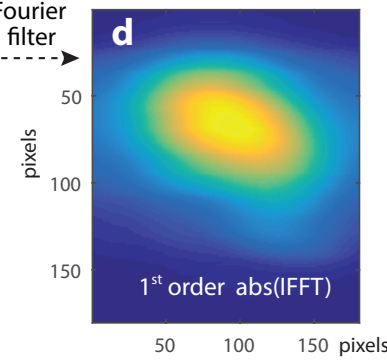

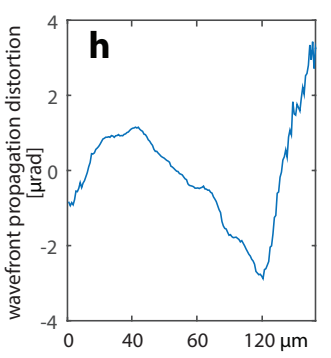

Figure 2. (a) Series of moiré interferograms used for XGI calibration. (b-h) Flowchart of the Fourier analysis algorithm of a moire pattern recorded with horizontally aligned line gratings. The wavefront propagation angle accounts for the vertical component only (see text).

Detailed description of the XGI based on a pattern of moiré interferometry can be found in references 2,3,8-10. Briefly, by rotating phase (or absorption) grating around the axis of the X-ray beam, a moiré fringe pattern is formed as shown in Figures 1 and 2. For the sake of convenient data recording we note that the resulting periodicity of the fringes is larger than the one formed merely by the phase grating, the magnifying factor is proportional to the angle $\theta$ depicted in Fig. 1. For a quantitative analysis Fig. 2a shows a calibration scan by recording the moiré patterns at varying angular $\theta$ orientations. Following the approach in Refs. 2, 10, 11, a full wavefront characterization can be determined from a single image such as seen in panel (b), once the transmission grating angle offset is derived from a rotation scan seen in panel (a). We note that by tracking the peak locations in horizontal and vertical dimensions from the respective first-order components in Fourier domain over the entire calibration scan, density curves are obtained. The shape of these curves readily identify if the wavefront propagation is diverging or converging, ${ }^{10}$ and indeed confirmed that all the wavefronts discussed below relate to focusing beams.

To further elucidate the wavefront analysis, Figure 2b-h shows a flowchart of the computational algorithm: a 
moiré interferogram in panel $\mathrm{b}$ is first-order Fourier filtered in panel $\mathrm{c}-\mathrm{d},{ }^{12}$ panels e-f illustrate the fringe phase recovery, and finally panels $g$-h retrieve the spherical and aspherical wavefront propagation angle, which allows to determine the wavefront radius of curvature (ROC). ${ }^{3,10,13}$ Below we apply this wavefront analysis to bendable toroidal mirror, beryllium compound refractive lenses (CRL) and zone plate (ZP) in order to characterize their beam transverse coherence.

\subsection{Bendable toroidal mirror}

a

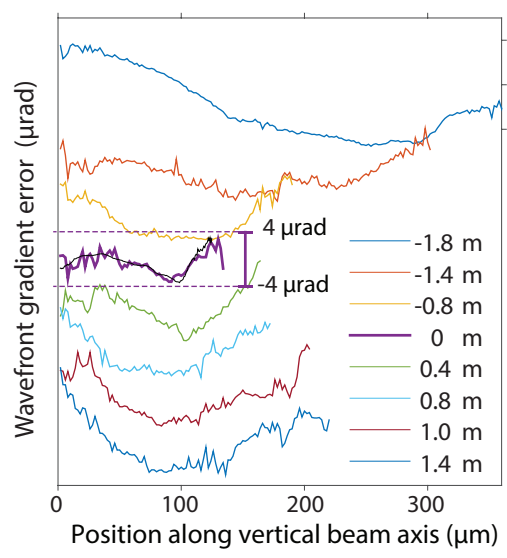

b

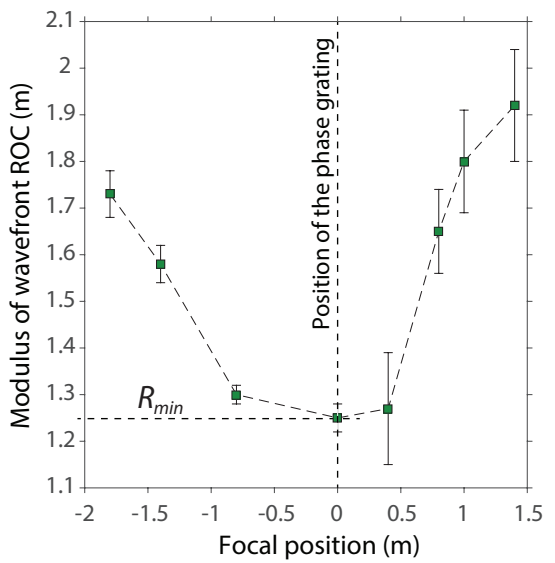

C

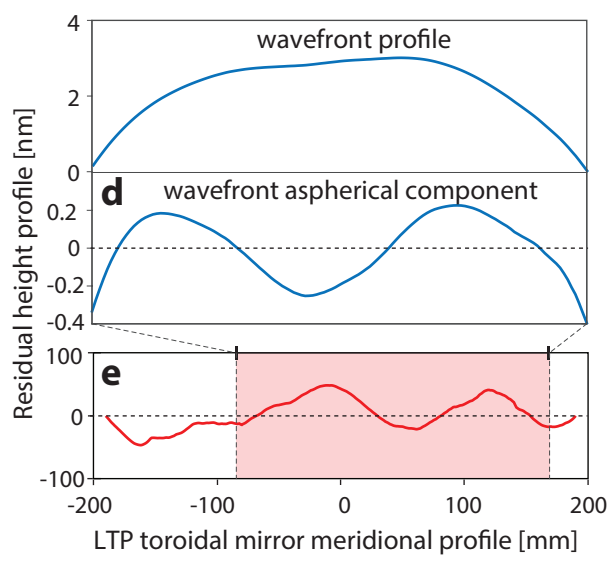

Figure 3. Wavefront analysis of a toroidal mirror. (a) Wavefront errors in the plane of the phase grating across the vertical beam axis. The different traces represent different mirror settings by moving the focus position along the beam direction through the plane of the grating, as depicted in 1top.(b) Derived modulus of the wavefront's radius of curvature (ROC) at the phase-grating versus the focus position along the beam axis. The minimum value is observed when the focus is situated close to the phase grating, with a $\pm 4 \mu \mathrm{rad}$ wavefront error when the focal spot is close to the phase grating plane (magenta line). For comparison, the wavefront error shown in Fig. 2, obtained before the mirror focus scan, is also plotted (dashed black line). (c-d) Reconstructed wavefront profile across the mirror surface and its corresponding spherical component, respectively. (d) Surface height error of the same mirror measured with an LTP, compared with the wavefront wavefront distortions measured at $8.3 \mathrm{keV}$. (see text).

An important aspect of the at-wavelength metrology is to characterize wavefront distortions associated with beam focus position manipulation. The SLS Optics beamline X05DA delivers in focused mode $\approx 10^{11}$ photons $/ \mathrm{sec}$ with a focus around $70 \times 140 \mu \mathrm{m}^{2}(\mathrm{FWHM} \mathrm{v} \times \mathrm{h}),{ }^{4}$ in agreement with the vertical projection of the focused wavefront seen in Fig. 2h. The X05DA nominal focus position is around $16.3 \mathrm{~m}$ from the source point $S$ (see Fig. 1), which is near the position of the first phase grating $P$ of our XGI moiré setup. The toroidal mirror bender allows for shifting the beam focus along the beam path, as schematically depicted in Fig. 1 top. For simplicity the zero focal position $F$ (the beamline nominal focus) is set at the position of the first grating $P$. Fig. 3a illustrates the wavefront propagation distortions as the beam focal position $F$ is nearing the position of the first grating $P$, stacked wavefronts in 2a show their evolution during such focal displacement. Comparing them to the $\pm 4 \mu \mathrm{rad}$ wavefront distortions at the nominal focus position we note that the bender introduces a factor two higher distortion e.g. at focal position $-1.8 \mathrm{~m}$.

In order to verify the moiré interferometry capabilities in terms of focus determination, we next discuss the related ROC changes during the focal scan seen in Fig. 3b. As expected from the simple geometrical considerations denoted in Fig. 1 top, approaching the focal position $F \rightarrow P$ we expect a $R O C \rightarrow 0$ convergence. We note that the minimum ROC $R_{\min }$ reflects correct nominal focal position of the beamline, however its magnitude of $\approx 1.2 \mathrm{~m}$ is significantly larger than expected. Moreover, by moving focal position further upstream, the ROC again increases, but error margins become significantly larger. From these experimental observations we conclude that our XGI setup is optimal for determining focal position at a minimum distance of $\approx 1.5 \mathrm{~m}$ in front of the first phase grating $P$. A possible explanation for this limitation is that as the focal point moves through the gratings, the experimental resolution significantly decreases. A possible explanation is at hand in Fig. 3b: 
the measured moiré patterns from focused beam are rather small (around $150 \times 150$ CCD pixels) compared to full area of the CCD chip $(1024 \times 1024)$. Consequently, the magnification of the detected focused beam is insufficient, which in turn reduces the resolution of the moire fringes and thus impose limitations in the wavefront analysis accuracy.

Another interesting aspect of XGI is the in situ monitoring of mirror surface quality. A mirror surface in front of the XGI setup allows to assess its height profile through a projection of the aspherical component of the wavefront angular phase distortion. ${ }^{3}$ Such an option calls for cross-checking the figure errors of focusing optics obtained for example with ex situ visible-light metrology based on interferometers or long-trace profilometers (LTP). ${ }^{14}$ At the X05DA beamline the analyzed wavefront height profiles seen in Fig. 3c-d, respectively, are limited by upstream slits, for clarity the incidence angle on the surface of the toroidal mirror is schematically depicted in Fig. 1. The peak-to-valley excursion of the wavefront aberrations is $\approx 0.2 \mathrm{~nm}$, which is a factor 2 higher than the monochromator height profile investigated with divergent beam without the toroidal mirror, ${ }^{3}$ meaning that the transverse coherence is exceeding the wavelength of the monochromatic beam $(\approx 0.14 \mathrm{~nm}$ at $8.3 \mathrm{keV}$ ). Moreover, comparing the height profiles measured with x-rays and visible light in Fig. 3d-e there is a firm evidence that the wavefront aspherical profile in panel (d) finds its analogue in the meridional LTP profile highlighted by red area in panel (e). Thus, the XGI proves to be complementary technique to ex situ LTP diagnostics with visible light.

\subsection{Beryllium compound refractive lenses}
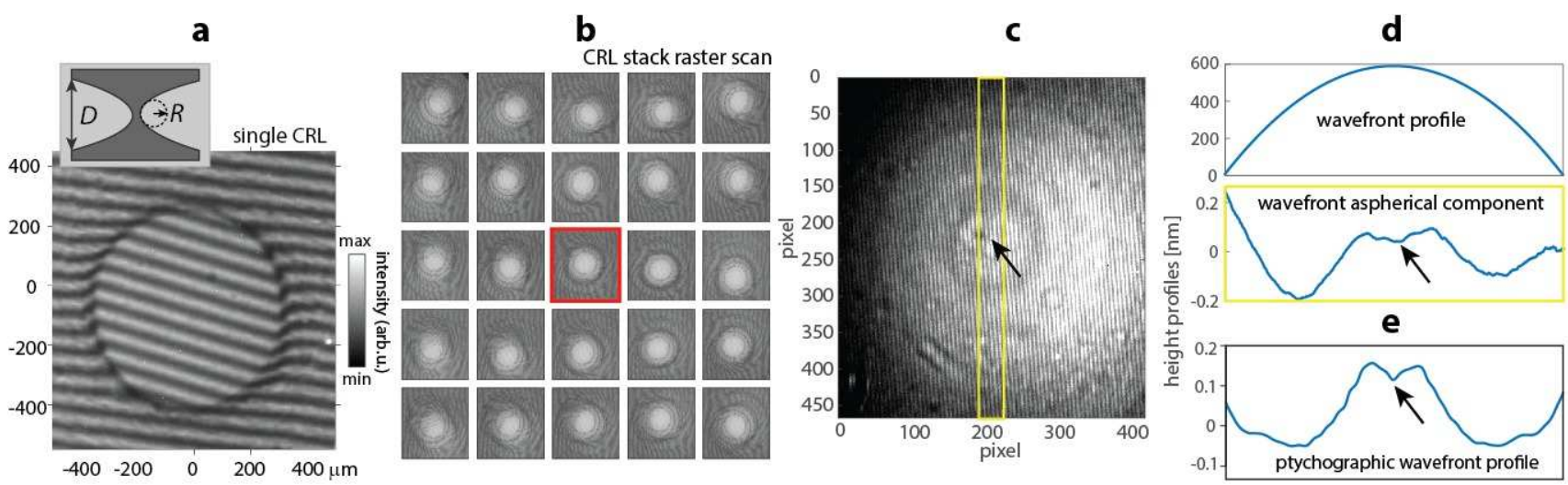

Figure 4. Wavefront analysis from Be CRLs. (a) Moiré interferogram from a single lens. (b) Series of moiré interferograms from a stack of $10 \mathrm{CRLs}$ in a motorized raster position scan, red frame indicates the optimized exit pupil position. Data were measured at ID13 Coherence beamline at DLS. (c-d) Vertical wavefront analysis of a CRL stack, measured at SLAC XPP beamline and compared with ptychographic data set from Ref. 15. Slanted arrows indicate a dip in the profile (see text).

Figure 4 summarize the moiré interferometric method to characterize the shape of a hard-x-ray wavefront from Be CRLs measured at DLS (panels a-b) and Linac Coherent Light Source (LCLS) XPP beamline (panels c-e), measured at 9.7 and $9.45 \mathrm{keV}$, respectively (see Table 1). Having in mind the limitations of our XGI setup discussed in section 2.1, the phase grating was located $2.35 \mathrm{~m}$ after the nominal beam focus. Fig. 4a shows the moiré interferogram with evident wavefront distortion related with the diameter $D$ of the CRL, schematically depicted in Fig. 4a. As expected, a more complex interferogram picture is obtained when the single-lens deformations pile up for the ensemble of 10 lenses. As seen in Fig. 4b, such a stack of lenses displays pronounced wavefront aberrations which require accurate alignment in order to optimize the size of the exit pupil free form these aberrations. The figure demonstrates such an alignment by means of an XGI raster scan of the whole stack mounted on adequate motorized stage. By optimizing the CRL stack position and angle with respect to the beam we find that the central interferogram indicated in red reflects the optimal stack alignment.

Figure 4c-d shows a more quantitative lens characterization from data at the LCLS XPP beamline, collected at the same distance from another system consisting of 20 lenses. Panels c-d show the measured wavefront spherical profile and its aspherical component which identified a vertical ROC of $2.5 \pm 0.3 \mathrm{~m}$, in good agreement 
with the installation $(2.3 \mathrm{~m})$. Because the waveform distortions with moiré interferometry are integrated over the selected region of interest (ROI), in order to account for the characteristic "sombrero-like" CRL waveform shape in the vertical direction, a narrow ROI denoted by yellow rectangle in Fig. 4c was considered.

Next we compare the aspherical wavefront components in Fig. 4d to scanning coherent x-ray diffraction imaging in panel e, known as ptychography. ${ }^{15-17}$ We emphasize that both techniques provide consistent qualitative and quantitative assessment of the vertical wavefront distortion around $\pm 0.2 \mathrm{~nm}$ peak-to-valley, which corresponds to $\lambda \approx 1.5$ wavelength at $9.45 \mathrm{keV}$. To further describe the wavefront distortions, the dip indicated by slanted arrows in Fig. 4 is related with the CRL apex radius of curvature $R$, schematically depicted in panel Fig. 4a. It turns out that a reduction of phase errors in individual refractive lenses is technically challenging mainly because the shape errors for individual lenses surfaces are within fabrication tolerances. However, the correction of residual spherical aberration of the whole stack by a corrective phase plate is feasible and can reduce the aberrations in the exit pupil down to $\approx 0.035 \mathrm{~nm} .{ }^{16}$

\subsection{Zone plates}

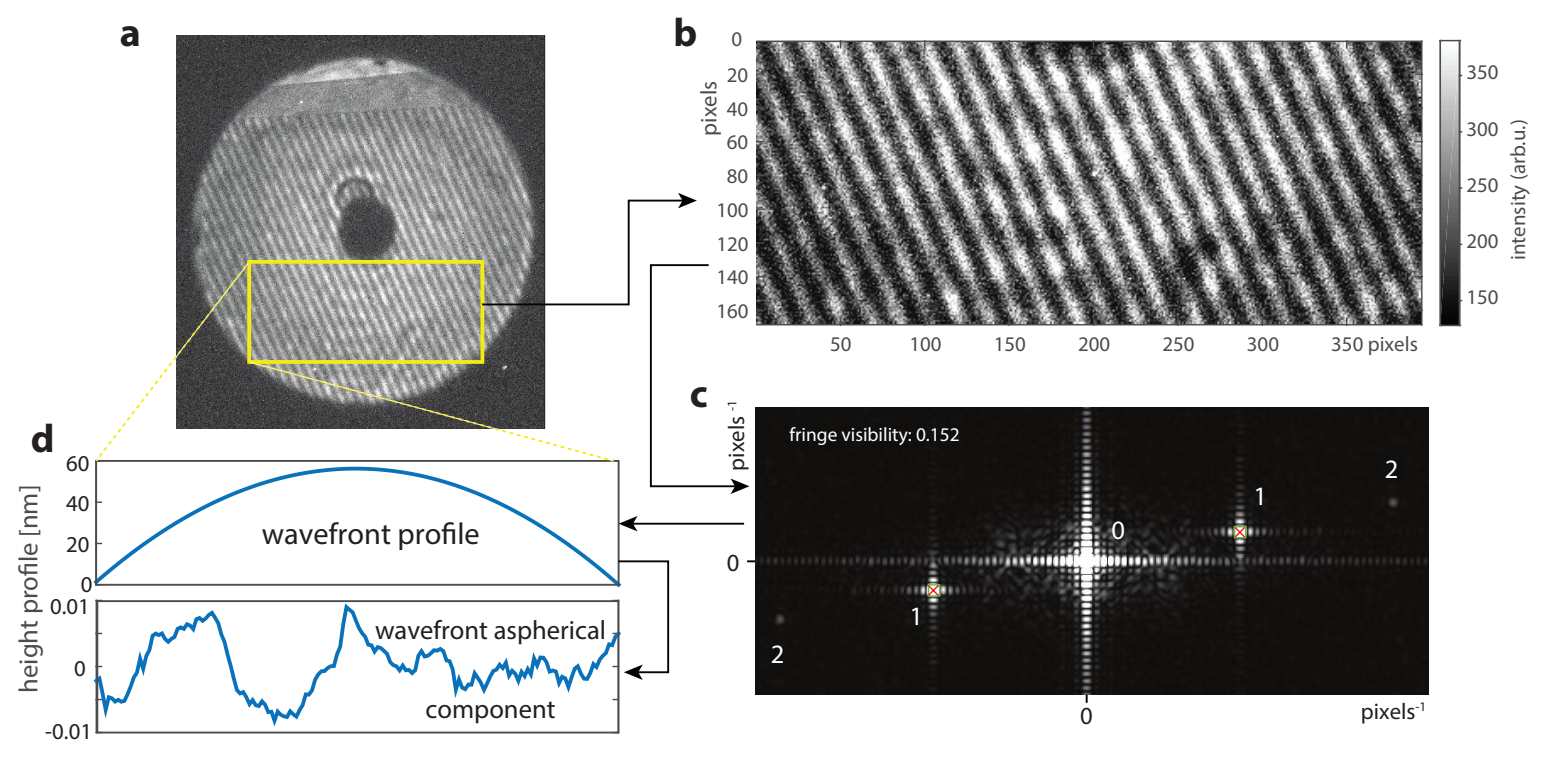

Figure 5. Wavefront analysis from a zone plate. (a-b) Moiré interferogram with selected ROI indicated by yellow frame, with corresponding Fourier domain data in (c) and resulting wavefront spherical and aspherical profiles in (d). Data were measured at ID13 Coherence beamline at DLS.

In all examples discussed beforehand the residual wavefront aberrations (i.e. after subtracting a spherical curvature) were considerably larger than the x-ray wavelength used for the measurements. To investigate the limits of XGI we undertook experiments at Diamond Light Source's Imaging and Coherence Beamline I13. This $250 \mathrm{~m}$ long hard $\mathrm{x}$-ray beamline combines an innovative optical beamline-layout with very high quality x-ray optics and advanced opto-mechanical controls to provide a smooth undisturbed x-ray wavefront with an exceptional large transversal coherence length much beyond $250 \mu \mathrm{m}$ at $9.7 \mathrm{keV}$ in both directions, i.e. horizontally and vertically. ${ }^{18}$ Fig. 5 shows the wavefront analysis behind the focus of a $\mathrm{ZP}$ with a diameter of $400 \mathrm{um}$ and an outermost zone width of $150 \mathrm{~nm}$. At $9.7 \mathrm{keV}$ the focal length of this zoneplate is $470 \mathrm{~mm}$.

To simplify the wavefront analysis a region of interest was selected to avoid the central stop of the zoneplate (see panel a). The high data quality of the fringe pattern (panel b), mainly due to the large coherence length, is confirmed by clearly observing the -2 nd and +2 nd orders in the Fourier domain (panel c) in comparison to 2(e), where only the first order is visible as expected from a lower coherence beamline. By deriving the wavefront from panel (c) and subtracting the best fitting quadratic-phase, one arrives at panel (d), showing residual wavefront distortions with an error margin of less than $\pm 0.01 \mathrm{~nm}$, which corresponds to $\approx \frac{1}{10}$ th of the wavelength. The 
focal position inferred from the incident vertical fringe phase is $2.3 \pm 0.3 \mathrm{~m}$, in agreement with the geometry of the overall setup.

Currently, with our single measurement, we cannot yet distinguish between contributions to the waveform distortions from the beamline optics (mirror, monochromator and vacuum windows) and the zoneplate. But because the residual aberrations are already below $\frac{\lambda}{10}$, it is clear that this beamline is very conducive to in situ x-ray metrology of beamline optics.

\section{CONCLUSION}

In conclusion, the moiré XGI instrument presented in this paper is a valuable at-at wavelength diagnostics tool for qualitative and quantitative, spatially resolved X-ray wavefront metrology at synchrotron radiation beamlines or XFEL endstations. Its compact and easy to move design have proven its merits for routine in situ X-ray wavefront characterization and at-wavelength metrology at synchrotron and XFEL facilities. Concerning determining the focal point of focusing optics, the experimental results shown from bendable mirror, CRL and ZP are in good agreement with the physical expectations. Such a performance is desirable for in situ characterising wavefront distortions during daily operation. These distortions may be caused by heat load effects or mechanical changes during focus adjustments. From this point of view, the XGI instrument can be considered a valuable diagnostic instrument, which is also highly appropriate for tuning adaptive optics.

\section{ACKNOWLEDGMENTS}

The authors thank to Yves Kayser for valuable discussions. We acknowledge Diamond Light Source for beamtime on the Diamond I13-1 branchline of the I13 Imaging and coherence beamline under the experiment number MT19521.

\section{REFERENCES}

[1] Kayser, Y., Rutishauser, S., Katayama, T., Kameshima, T., Ohashi, H., Flechsig, U., Yabashi, M., and David, C., "Shot-to-shot diagnostic of the longitudinal photon source position at the spring-8 angstrom compact free electron laser by means of x-ray grating interferometry," Opt. Lett. 41, 733-736 (Feb 2016).

[2] Wang, H., Sawhney, K., Berujon, S., Ziegler, E., Rutishauser, S., and David, C., "X-ray wavefront characterization using a rotating shearing interferometer technique," Opt. Express 19, 16550-16559 (Aug 2011).

[3] Kayser, Y., David, C., Flechsig, U., Krempaský, J., Schlott, V., and Abela, R., "X-ray grating interferometer for in situ and at-wavelength wavefront metrology," Journal of Synchrotron Radiation 24(1), 150-162 (2016).

[4] Flechsig, U., Jaggi, A., Spielmann, S., Padmore, H., and MacDowell, A., "The optics beamline at the Swiss Light Source," Nuclear Instruments and Methods in Physics Research Section A: Accelerators, Spectrometers, Detectors and Associated Equipment 609(2), 281 - 285 (2009).

[5] Rivers, M. L., Garrett, R., Gentle, I., Nugent, K., and Wilkins, S., "areaDetector: Software for 2D Detectors in EPICS," in [Medical Imaging: Image Processing], Loew, M. H., ed., AIP Conf. Proc. 1234, 51-54 (2010).

[6] Rutishauser, S., Zanette, I., Weitkamp, T., Donath, T., and David, C., "At-wavelength characterization of refractive x-ray lenses using a two-dimensional grating interferometer," Applied Physics Letters 99(22), 221104 (2011).

[7] Engelhardt, M., Baumann, J., Schuster, M., Kottler, C., Pfeiffer, F., Bunk, O., and David, C., "Inspection of refractive x-ray lenses using high-resolution differential phase contrast imaging with a microfocus x-ray source," Review of Scientific Instruments 78(9), 093707 (2007).

[8] Pfeiffer, F., Bunk, O., Schulze-Briese, C., Diaz, A., Weitkamp, T., David, C., van der Veen, J. F., Vartanyants, I., and Robinson, I. K., "Shearing interferometer for quantifying the coherence of hard x-ray beams," Phys. Rev. Lett. 94, 164801 (Apr 2005).

[9] Weitkamp, T., Nöhammer, B., Diaz, A., David, C., and Ziegler, E., "X-ray wavefront analysis and optics characterization with a grating interferometer," Applied Physics Letters 86(5), 054101 (2005).

[10] Rutishauser, S., X-ray grating interferometry for imaging and metrology, PhD thesis, ETH ZURICH (2013). Diss. ETH No. 20939. 
[11] David, C., Rutishauser, S., Sprung, M., Zanette, I., and Weitkamp, T., "X-ray grating interferometry applications in metrology and wave front sensing," AIP Conf. Proc. ed. by A. Momose and W. Yashiro (1), 1466 (2012).

[12] Takeda, M., Ina, H., and Kobayashi, S., "Fourier-transform method of fringe-pattern analysis for computerbased topography and interferometry," J. Opt. Soc. Am. 72, 156-160 (Jan 1982).

[13] Rutishauser, S., Samoylova, L., Krzywinski, J., Bunk, O., Grönert, J., Sinn, H., Cammarata, M., Fritz, D. M., and David, C., "Exploring the wavefront of hard x-ray free-electron laser radiation," Nature Communications 3, $947 \mathrm{EP}$ - (Jul 2012). Article.

[14] Flechsig, U., Jaggi, A., Krempaský, J., Spielmann, S., and Thominet, V., "The upgraded LTP-V at SLS," Nuclear Instruments and Methods in Physics Research Section A: Accelerators, Spectrometers, Detectors and Associated Equipment 710, 13 - 16 (2013). The 4th international workshop on Metrology for X-ray Optics, Mirror Design, and Fabrication.

[15] Seiboth, F., Schropp, A., Scholz, M., Wittwer, F., Rödel, C., Wünsche, M., Ullsperger, T., Nolte, S., Rahomäki, J., Parfeniukas, K., Giakoumidis, S., Vogt, U., Wagner, U., Rau, C., Boesenberg, U., Garrevoet, J., Falkenberg, G., Galtier, E. C., Lee, H. J., Nagler, B., and Schroer, C. G., "Aberration correction for hard x-ray focusing at the nanoscale," Proc.SPIE 10386, 10386 - 10386 - 10 (2017).

[16] Seiboth, F., Schropp, A., Scholz, M., Wittwer, F., Rödel, C., Wúnsche, M., Ullsperger, T., Nolte, S., Rahomá, J., Parfeniukas, K., Giakoumidis, S., Vogt, U., Wagner, U., Rau, C., Boesenberg, U., Garrevoet, J., Falkenberg, G., Galtier, E. C., Ja Lee, H., Nagler, B., and Schroer, C. G., "Perfect x-ray focusing via fitting corrective glasses to aberrated optics," Nature Communications 8, 14623 EP - (Mar 2017). Article.

[17] Seiboth, F., Wittwer, F., Scholz, M., Kahnt, M., Seyrich, M., Schropp, A., Wagner, U., Rau, C., Garrevoet, J., Falkenberg, G., and Schroer, C. G., "Nanofocusing with aberration-corrected rotationally parabolic refractive X-ray lenses," Journal of Synchrotron Radiation 25, 108-115 (Jan 2018).

[18] Wagner, U., Parson, A., and Rau, C., "Coherence Length and Vibrations of the Coherence Beamline I13 at the Diamond Light Source," Journal of Physics: Conference Series 849(1), 012048 (2017). 